progress of individual medical and surgical teams throughout the implementation timeline (Figure 1). Our secondary outcome measure was the percentage of patients discharged within 2 hours of meeting their medical discharge goals.

Results The percentage of patients whose discharge process included medical goals increased from $25 \%$ to $76 \%$ in 3 years. The progress of individual teams is displayed in Figure 2. For patients who followed the process, the percentage of patients discharged within 2 hours of meeting medical discharge goals increased from $42 \%$ to $85 \%$ (Figure 3); however, this overestimates success, as patients are only included in this measure if all parts of the process are followed.

Conclusions We successfully spread discharge efficiency based upon medical goals to a majority of hospitalised acute care patients; however, we continue to identify improvement opportunities with process adherence.

\section{EVALUATION OF A MULTIDISCIPLINARY CANCER CLINIC: IMPROVING TIME TO ONCOLOGY ASSESSMENT AND TREATMENT FOR PATIENTS WITH NEW LUNG CANCER}

${ }^{1}$ Christopher Stone, ${ }^{2}$ Erin Brown, ${ }^{1}$ Mihaela Mates, ${ }^{1}$ Conrad Falkson, ${ }^{1}$ Timothy Owen, ${ }^{1}$ Allison Ashworth, ${ }^{1}$ Aamer Mahmud, ${ }^{1}$ Christopher Parker, ${ }^{1}$ Andrew Robinson, ${ }^{1}$ Genevieve Digby. ${ }^{1}$ Kingston Health Science Centre and Queen's University, Canada; ${ }^{2}$ South East Regional Cancer Program, Canada

\subsection{6/bmjoq-2017-IHI.12}

Background Delays in the management of lung cancer (LC) are associated with inferior outcomes. Multidisciplinary cancer clinics (MDCC) can improve timeliness and quality of care.

Objectives Decrease time from LC diagnosis to oncology assessment from 13 to 3 days, and to treatment from 30 to $<20$ days, within 6 months.

Methods We implemented a weekly MDCC, involving Respirologists, Medical Oncologists (MO) and Radiation Oncologists (RO), where patients with new LC diagnoses received concurrent oncology consultation. We retrospectively analysed data pre-MDCC (November 2016 - February 2017) and prospectively for improvements (February - July 2017). Improvement cycles included MDCC clinic launching and a debriefing/troubleshooting meeting. Data are reported as $\mathrm{n}(\%)$, and means as per Statistical Process Control XmR(i) charts.
Results 117 patients (44 pre-MDCC, 73 post-MDCC) were analysed. Most patients had stage $4(44,37.6 \%)$ or stage 1 LC $(32,27 \%)$. All patients saw Respirology, in addition to MO $(85,72.6 \%)$, RO $(113,96.6 \%)$, or both $(83,71.0 \%)$. The proportion of treated patients was unchanged pre- vs. post-MDCC $(88.6 \%, 85.4 \%)$. Mean days from diagnosis to oncology assessment decreased from 14.3 to 5.0 days. Time from diagnosis to first treatment decreased from 39.8 to 27.2 days after the first improvement cycle, and to 18.1 days after the second improvement cycle (Figure 1), with less variation in time to treatment after improvement events.

Conclusions MDCC shortens time from LC diagnosis to oncology assessment and treatment. Time to treatment improved more than time to oncology assessment, suggesting the improvement is likely related to benefits beyond just faster oncology assessment.

\section{REDUCING CARDIAC ARRESTS IN AN ACUTE MEDICAL UNIT}

Calum McGregor, Sanjiv Chohan, Jonathan O'Reilly. NHS Lanarkshire, UK

\subsection{6/bmjoq-2017-IHI.13}

Background Cardiac arrests are often preceded by a period of physiological deterioration. Preventing cardiac arrests depends on reliable recognition of, and response to, those deteriorations. Our Acute Medical Unit was identified as having the highest number of cardiac arrests in the hospital in 2013/ 2014. Our baseline cardiac arrest was 4.3/1000 (October 2014 - February 2016).

Objectives The aim was to reduce our unit's cardiac arrest rate by over $50 \%$.

Methods Process mapping exercises identified unreliable processes in the recognition and response to deteriorating patients. Pareto chart analysis (Figure 1) identified hypoxia as the most commonly missed cause of deterioration within the unit. The model for improvement and rapid cycle tests of change were used to develop standardise key clinical processes. Innovative multi-disciplinary learning from what went well, called 'Save of the Month', helped to identify good practice and develop pride in work.

Results The cardiac arrest rate showed 63\% reduction from the baseline period; 4.3/1000 (October 2014 to February 2016) to

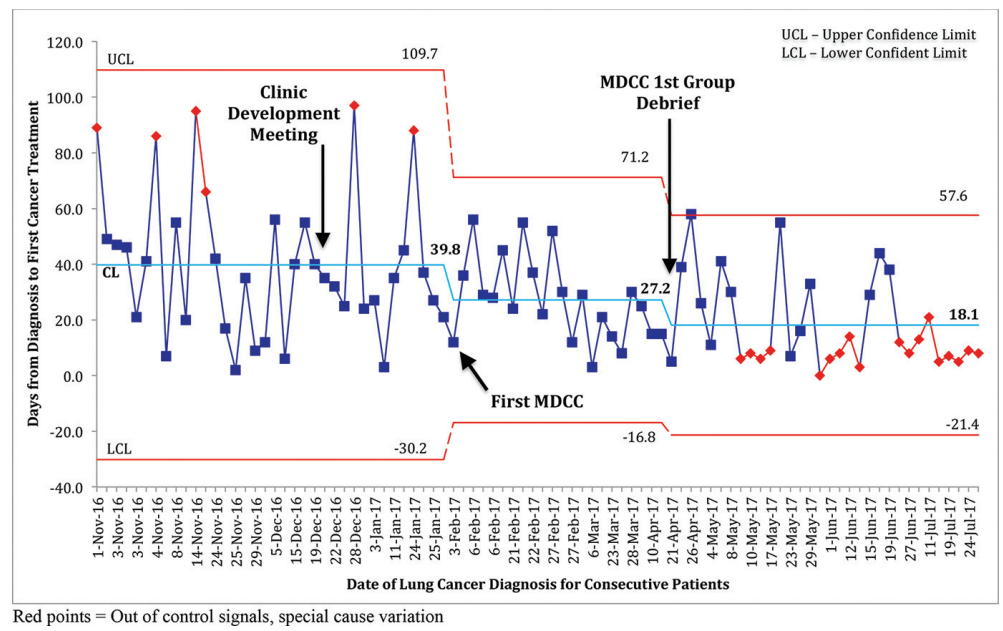

Abstract 916 Figure 1 Effect of MDCC on time from lung cancer diagnosis to first cancer treatment 


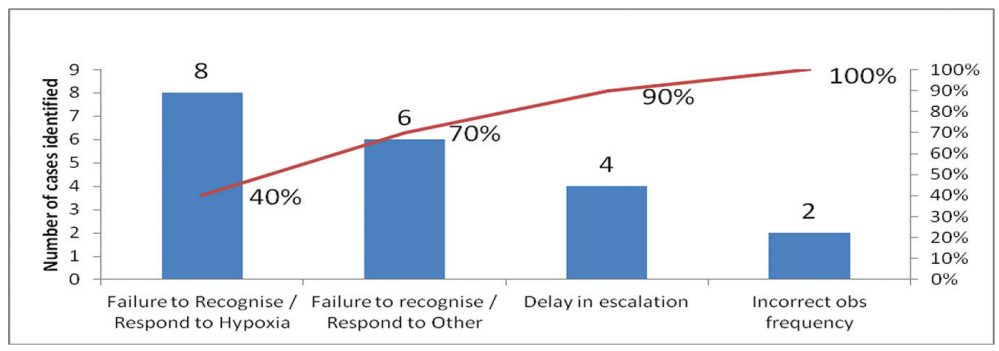

Abstract 920 Figure 1 Case note review identification of sub-optimal care

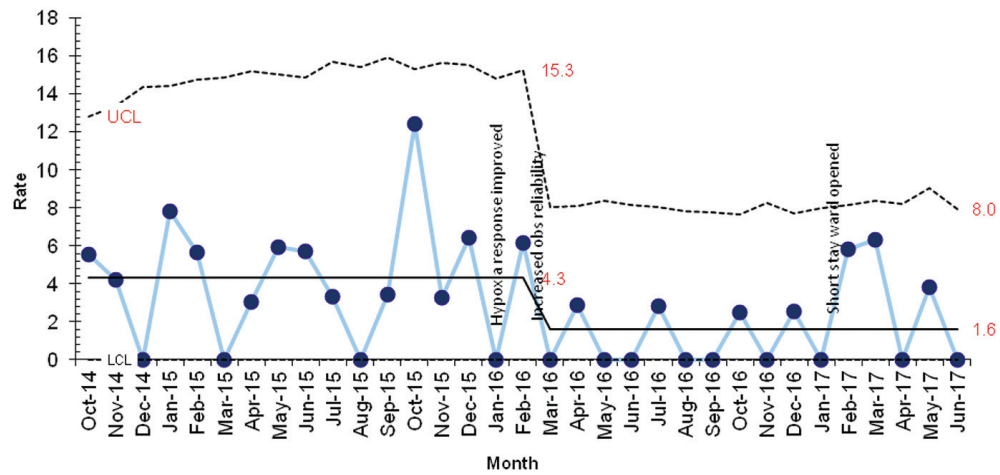

Abstract 920 Figure 2 Cardiac arrest rate per 1000 deaths/discharges SPC-U chart

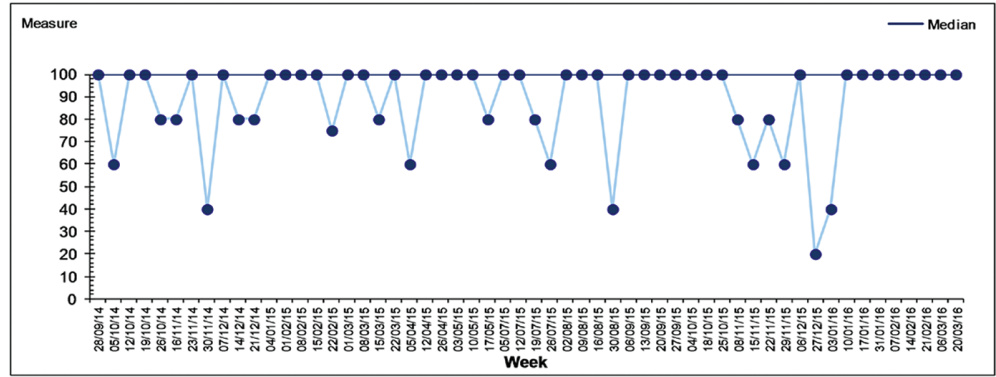

Abstract 920 Figure 3 Reliable implementation of observation bundle

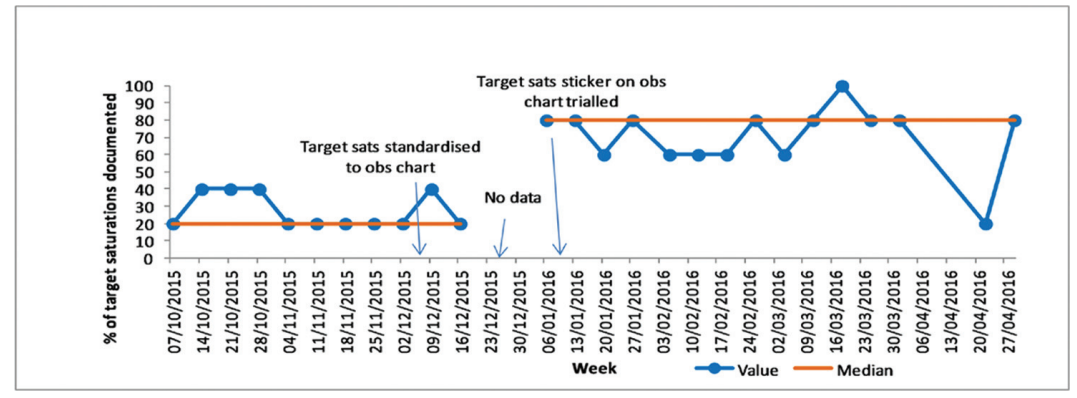

Abstract 920 Figure 4 Target saturations documention on clinical obs chart

1.6/1000 (March 2016 to June 2017). 11580 patients were included in this time period (Figure 2). The cardiac arrest reduction was associated with significant improvements in the following process measures when run chart rules are applied: clinical observation bundle completion, documentation of target oxygen saturations, identification of hypoxia and completion of structured response to hypoxia (Figures 3-7).
Conclusions Multi-disciplinary learning from what went well can help address psychological barriers to change. This project enabled a multi-disciplinary frontline team to engage in quality improvement, identify their own local problems and test their solutions scientifically. 